ORIGINAL ARTICLE

\title{
Predictors of mortality in mechanically ventilated patients
}

\author{
T D Sudarsanam, L Jeyaseelan, K Thomas, G John
}

Postgrad Med J 2005;81:780-783. doi: 10.1136/pgmj.2005.033076

See end of article for authors' affiliations .....................

Correspondence to: Dr T D Sudarsanam, Department of Medicine 2, Christian Medical College and Hospital, Vellore 632004, Tamilnadu, India; thambu@cmcvellore.ac.in

Submitted 27 January 2005 Accepted 16 April 2005
Objectives: There are scarce data from India validating scoring systems used to predict outcome in patients requiring mechanical ventilation. This study prospectively compared the organ system failure (OSF), the acute physiology and chronic health evaluation (APACHE) II, and the APACHE III, scores on patients requiring mechanical ventilation in the medical intensive care unit, to predict outcome.

Methods: 200 consecutive patients requiring mechanical ventilation in a medical intensive care unit were recruited. OSF, APACHE II, and APCHE III scores were calculated at admission and daily for one week or until discharge in all patients. Other variables recorded include age, sex, diagnosis, oxygen therapy before ventilation, complications on ventilation, duration in hospital before ventilation, duration of ventilation, type of respiratory failure, alveolar arterial oxygen gradient, P/F ratio, use of tracheostomy, time on ventilator before tracheostomy, muscle relaxant used, fluid balance, inotrope support. Logistic regression analysis and area under the curve were computed to determine which variables independently predict outcome.

Results: Of the 200 patients, at discharge 143 patients (71.5\%) had died. The factors that independently predicted outcome among these patients on mechanical ventilation were the type of respiratory failure (type I) OR=2.7 ( $p=0.02)$, the use of inotropes OR $2.4(p=0.04)$, and the APACHE II score OR= 1.8 $(p=0.008)$ for every five point increase in APACHE II score.

Conclusions: Type 1 respiratory failure, the use of inotropes, and the APACHE II score measured at admission are significant independent predictors of mortality in the patients on mechanical ventilation.
M echanical ventilation is an essential life support given to many patients in the intensive care unit (ICU). ${ }^{1}$ Scoring systems have been developed to help the physician predict mortality in patients requiring critical care. ${ }^{2}$ Patients admitted in the ICUs and the technological care that can be provided in the developing world is substantially different from that noted in the developed countries. There are scarce data validating these scoring systems in patients requiring mechanical ventilation from India. We prospectively compared the three scoring systems to predict clinical outcome: the organ system failure (OSF), the acute physiology and chronic health evaluation (APACHE) II, and the APACHE III scores along with other variables that could possibly have a relation to outcome in patients requiring mechanical ventilation in the medical intensive care unit (MICU) in a tertiary care centre in India.

\section{METHODS}

The study was conducted in a tertiary teaching hospital in South India between March 1997 and March 1998. Two hundred consecutive patients admitted in the MICU requiring mechanical ventilation were recruited. The scores obtained in OSF, ${ }^{3}$ APACHE $\mathrm{II}^{4}$ and APACHE $\mathrm{III}^{5}$ were calculated at admission and daily for one week or until discharge in all patients. The other variables that were recorded were age, sex, diagnosis at admission, oxygen therapy before ventilation, complications on ventilation, duration in hospital before ventilation, duration of mechanical ventilation, type of respiratory failure, alveolar arterial oxygen gradient, $\mathrm{P} / \mathrm{F}$ ratio $\left(\mathrm{PaO}_{2} / \mathrm{FiO}_{2}\right)$, use of tracheostomy, time on ventilator before tracheostomy, muscle relaxant used, fluid balance, inotrope support, clinical outcome (dead/ survived).

\section{Statistical analysis}

SPSS for Windows version 8 was used for analysis. Logistic regression analysis was done with clinical outcome as dependent variable and other measured variables, which were noted to have significant $(p<0.05)$ association in univariate analysis. The scores obtained by the three scoring systems were compared using the receiver operating characteristics (ROC) methods. ${ }^{6}$ The likelihood ratio (LR) was calculated for predicting outcome at different cut off points for OSF, APACHE II, and APACHE III. Area under the curve (AUC) was calculated for the three scores and compared statistically in a paired manner. Odds ratios of scoring systems with cut off of five were calculated to make clinical interpretation easier.

\section{RESULTS}

During the 12 month study period 483 patients were admitted to the MICU. The overall case fatality for patients admitted in the MICU was 148 of 483 (30.6\%). Of the 483 patients 283 patients did not require mechanical ventilation; five $(1.7 \%)$ of these patients died.

Two hundred patients were enrolled in the study. Sixty three $(28.5 \%)$ were women and $137(68.5 \%)$ were men. Of the 200 patients $143(71.5 \%)$ died and 57 (28.5\%) survived.

Table 1 shows the admission diagnosis for the study patients.

Among the variables entered into the univariate analysis (tables 2 and 3) the following were found to predict mortality in mechanically ventilated patients: type of respiratory failure, use of inotrope support, age, duration in hospital before mechanical ventilation, alveolar arterial oxygen pressure gradient, and APACHE II score. The correlation coefficient between APACHE II and APACHE III scores was 0.8 and 0.63 between APACHE II and OSF scores. The six variables were entered into the logistic regression analysis.

Abbreviations: ICU, intensive care unit; MICU, medical intensive care unit; APACHE, acute physiology and chronic health evaluation; OSF, organ system failure; ROC, receiver operating curve; AUC, area under curve 
Table 1 Admission diagnosis

\begin{tabular}{llll}
\hline Diagnosis & Percentage of patients & Number of patients & Mortality (number) \\
\hline Respiratory disease & 12 & 24 & 18 \\
Renal disease & 8 & 16 & 12 \\
Cardiovascular disease & 6 & 12 & 10 \\
Gastrointestinal disease & 7.5 & 15 & 11 \\
CNS disease & 16.5 & 33 & 28 \\
Infectious disease & 9.5 & 19 & 16 \\
Poisoning & 28.5 & 57 & 26 \\
Malignancy & 5.5 & 11 & 11 \\
Autoimmune disease & 6.5 & 13 & 11 \\
Total & 100 & 200 & 143 \\
\hline
\end{tabular}

Table 2 Univariate analysis (variables compared using the $t$ test)

\begin{tabular}{|c|c|c|c|c|}
\hline Variable & Overall mean & Alive mean (SD) & Died mean (SD) & p Value \\
\hline Age (y) & 41 & $34(16)$ & $43(18)$ & 0.001 \\
\hline $\begin{array}{l}\text { Duration in hours in hospital } \\
\text { before ventilation }\end{array}$ & 66 & $15(35)$ & $86(206)$ & 0.012 \\
\hline $\begin{array}{l}\text { Duration of mechanical } \\
\text { ventilation }(h)\end{array}$ & 116 & $168(124)$ & $95(123)$ & $<0.001$ \\
\hline $\begin{array}{l}\text { Alveolar-arterial (A-a) oxygen } \\
\text { gradient }\end{array}$ & 391 & $350(156)$ & $408(145)$ & 0.015 \\
\hline $\mathrm{P} / \mathrm{F}$ ratio & 2.9 & $3.3(2.0)$ & $2.7(4.4)$ & 0.33 \\
\hline Fluid balance in litres & +4.2 & $+6.3(6.9)$ & $+3.4(4.8)$ & 0.001 \\
\hline $\begin{array}{l}\text { Time before tracheotomy (h) } \\
\text { (evaluated for only } 62 \text { patients) }\end{array}$ & 65 & $110(86)$ & $32(55)$ & $<0.001$ \\
\hline
\end{tabular}

Table 3 Univariate analysis (variables compared using $\chi^{2}$ test with Yates's continuity correction)

\begin{tabular}{|c|c|c|c|c|c|c|}
\hline Variable & & Alive & Died & p Value & Odds ratio & $95 \% \mathrm{Cl}$ \\
\hline \multirow[t]{2}{*}{ Inotrope support } & Yes & 13 & 80 & $<0.001$ & 4.36 & 2.06to 9.39 \\
\hline & No & 44 & 62 & & & \\
\hline \multirow[t]{2}{*}{ Type of respiratory failure } & Type 1 & 14 & 77 & $<0.001$ & 3.58 & 1.72 to 7.56 \\
\hline & Type II & 43 & 66 & & & \\
\hline \multirow[t]{2}{*}{ Sex } & Male & 43 & 94 & 0.24 & 0.62 & 0.29 to 1.32 \\
\hline & Female & 14 & 49 & & & \\
\hline \multirow[t]{2}{*}{ Use of tracheotomy } & Yes & 26 & 36 & 0.01 & 0.40 & 0.2 to 0.8 \\
\hline & No & 31 & 107 & & & \\
\hline
\end{tabular}

The duration of mechanical ventilation and the cumulative fluid balance are two variables that were found significant on the univariate analysis, but were not entered into the multivariate analysis as they cannot be determined before or at the start of ventilation and hence cannot be used to predict outcome.

The results for the three scores done after a week were similar to those at admission and hence the time trend analysis is not presented.

\section{Results of the logistic regression analysis: (table 4)}

In the analysis APACHE II was found to be the only predictor of mortality among the three scores. As the analysis showed that there was close correlation between the APACHE II, APACHE III, and OSF scores the regression analysis was repeated after removing the APACHE II score and the APACHE III score was found to be an independent predictor. Then the analysis was repeated after removing the APACHE III score and the OSF score was seen to be an independent predictor of mortality.

The final logistic model included type of respiratory failure, use of inotropic support, and APACHE II as the APACHE II score had the highest level of association.
Table 5 looks at the sensitivity, specificity, and likelihood ratios for the APACHE II scoring system at different cut off points to predict outcome.

Figure 1 shows the receiver operating characteristic curve for the three scoring systems.

Complications that were seen in our patients included tube block in 19 patients $(9.5 \%)$, nosocomial pneumonia in 53 $(26.5 \%)$, nosocomial sepsis 3 ( $1.5 \%)$, and other complications in $4(2 \%)$. Altogether 119 patients had no complications related to mechanical ventilation.

\begin{tabular}{lll} 
Table 4 & Logistic regression analysis & \\
\hline Variable & Odds ratio (95\% CI) & p Value \\
\hline Type of respiratory failure & $2.69(1.20$ to 6.00$)$ & 0.016 \\
Use of inotropes * for a five & $2.39(1.10$ to 5.30$)$ & 0.035 \\
APACHE II score * & 1.80 (1.67 to 1.87$)$ & 0.008 \\
point increase in score & & \\
\hline
\end{tabular}

The following variables were not found to be independent predictors of mortality: duration in hours in hospital before ventilation $(p=0.09)$; alveolar-arterial oxygen $(A-a)$ gradient $(p=0.86)$; age $(p=0.55)$. 
Table 5 Apache II score: sensitivity, specificity, and likelehood ratios

\begin{tabular}{llll}
\hline Score & Sensitivity & Specificity & Likelihood ratio \\
\hline 3 & 99.3 & 0.00 & 0.99 \\
8 & 99.3 & 5.4 & 1.05 \\
11 & 97.9 & 12.5 & 1.12 \\
14 & 95.0 & 23.2 & 1.24 \\
15 & 95.0 & 27.0 & 1.30 \\
20 & 86.0 & 50.0 & 1.72 \\
22 & 78.0 & 61.0 & 2.00 \\
23 & 73.9 & 68.0 & 2.31 \\
24 & 70.0 & 71.0 & 2.41 \\
25 & 62.4 & 75.0 & 2.50 \\
26 & 59.6 & 78.6 & 2.78 \\
27 & 51.8 & 78.6 & 2.41 \\
28 & 44.0 & 83.9 & 2.74 \\
31 & 27.5 & 94.6 & 5.12 \\
36 & 17.0 & 98.2 & 9.55 \\
37 & 14.8 & 100.0 & Infinity \\
\hline
\end{tabular}

\section{DISCUSSION}

Scoring systems primarily developed for estimating prognosis in critically ill patients have not been well validated in mechanically ventilated patients. In an earlier study ${ }^{2}$ on 500 consecutive patients from India admitted in the MICU, modified OSF score was found superior to the APACHE II score in predicting mortality. This study was done on Indian patients to determine the usefulness of these scoring systems on our mechanically ventilated patients. This is important considering the limitation of resources and demand for ICU beds in most hospitals.

The data show that $41 \%$ of patients admitted to the MICU needed mechanical ventilation and the outcome of those not requiring ventilatory support was significantly better than those requiring ventilatory support-mortality $1.7 \%$ compared with $71.5 \%(\mathrm{p}<0.001)$.

The analysis of mortality in the diagnostic categories showed the least mortality in the patients admitted for

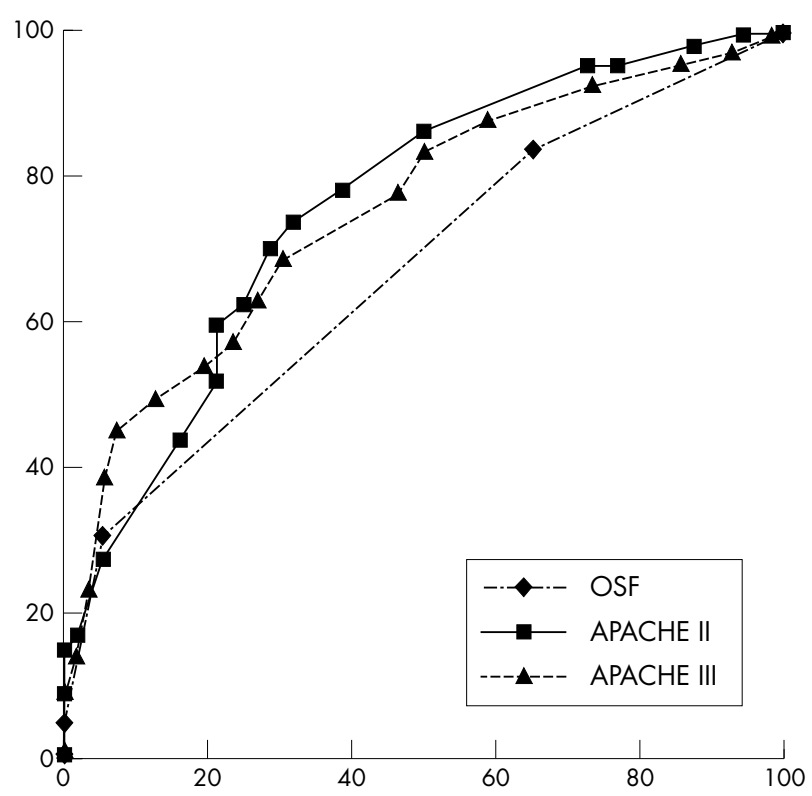

Figure 1 OSF, APACHE II, and APACHE III ROC curves. The area under the curve (AUC) for the three scores (APACHE II, APACHE III, and OSF) were $0.756 \mathrm{SE}(0.037), 0.7555$ (0.038), and $0.674(0.040)$ respectively. The difference between the APACHE II and III scores AUC was not significant $p=0.50$. poisoning $(46 \%)$, and highest in patients having a malignancy $(100 \%)$, findings similar to those reported earlier ${ }^{8}$ questioning the use of mechanical ventilation in patients whose underlying disease is not treatable; however most of these patients were admitted to the ICU for coexisting illnesses-mostly infections that were hoped to be reversible.

Studies done on patients with type II respiratory failure show that older patients with lower mean arterial pressures, higher blood urea, ${ }^{9}$ poorer pre-morbid condition, ${ }^{10-12}$ patients requiring ventilation within first 24 hours, ${ }^{13}$ patients with lower serum albumin, in cor pulmonale with lower $\mathrm{FEV}_{1}$ were found to have poorer prognosis. These variables, however, were not shown to independently predict outcome in our study, although most are included in the three scoring systems. Among patients with type I respiratory failure, sepsis, multi-organ system failure, cardiac failure, worsening renal function, ${ }^{14}{ }^{15}$ higher bronchoalveolar fluid interleukin 8 concentrations, ${ }^{16}$ a longer duration of mechanical ventilation, medically ill patients with sepsis and multiple transfusions as compared with trauma patients with the above comorbidities $^{17}$ were all found to have an adverse effect on the outcome. The duration of mechanical ventilation cannot be used prospectively to predict outcome, while the other factors are mostly incorporated in the APACHE II and III scores. Gupta et al have shown an APACHE III score of $>57$ and a SAPS II score of $>39$ predicted a poorer outcome. ${ }^{18}$

Logistic regression analysis shows that the factors that had prognostic significance on outcome were type I respiratory failure, use of inotropes, and the APACHE II score. Age, duration of mechanical ventilation, alveolar oxygen gradient, and $\mathrm{PaO}_{2} / \mathrm{FiO}_{2}$ gradient, although significant on univariate analysis were not found to predict outcome independently on multivariate analysis.

Patients with type I respiratory failure were found to have a higher mortality (40\%) as compared with 17\% with type II failure. These findings have been seen in earlier studies, ${ }^{9}$ the mortality rate for type II respiratory failure ranging from $9 \%$ to $50 \%{ }^{12}$ and from $54 \%{ }^{14}$ to $72 \%{ }^{20}$ in type I respiratory failure, showing that parenchymal lung damage predicts a poorer outcome for patients on mechanical ventilation. The reason for this difference could be because patients with type II failure are mostly those with poisoning and they have a good prognosis because they have a reversible illness, they have no underlying lung damage, and they do not have multiorgan system failure.

The use of inotrope support during mechanical ventilation is an independent predictor of outcome of these patients. This is probably because these patients have cardiovascular system failure and are unable to cope with the additional demands of a critical illness. Continuous monitoring of all ICU patients did not disclose any arrythmias in these patients. The common inotropes used in our ICU were dopamine, dobutamine, and adrenaline (epinephrine).

All three scoring systems were found to predict outcome. The APACHE II score as seen in the logistic regression analysis being the best of three systems in this regard. A five point increase in APACHE II score associated with a 1.8 times increase in mortality. A similar trend was seen in the ROC curves as well, although the difference was not statistically significant. In a recent study Nevins and Epstein ${ }^{21}$ have also shown that the APACHE II associated comorbidities predicted a poorer outcome for COPD patients requiring mechanical ventilation. The reasons for the APACHE III score not performing as well as the APACHE II score are not clear. The possible reasons could be (1) arterial $\mathrm{pH}$ is not included in the APACHE III scoring system. This has been shown to have a significant predictive value $\mathrm{e}^{22-24}$ especially as many of our patients have sepsis and (2) additional variables in the APACHE III such as bilirubin and glucose may not have any 
bearing on the population studied but seem to decrease its predictive value in our patient population. The APACHE II scores are much easier to calculate and apply than the APACHE III scores. The reason for comparing both was that if both were found equivalent or APACHE II found better than APACHE III (as was the case) then this easier to calculate scoring system (APACHE II) would be adapted for use.

As seen in table 5 the APACHE II score of 22 can be used as a cut off score in the ICU setting to predict mortality. The analysis shows that this score has a likelihood ratio of 2.00, a positive predictive value of $83 \%$, and a negative predictive value of $52 \%$. Thus this score can be used to "rule in" a higher mortality. In the ICU context this score, which has a comparatively high sensitivity in predicting mortality, will be useful to guide the physician in decisions prognosis.

In conclusion we have noted that the type of respiratory failure, the use of inotropes, and APACHE II score, are the important independent predictive factors that determine outcome of patients on mechanical ventilation. These data will be a valuable aid to clinicians and relatives of patients on mechanical ventilation in deciding on the probable outcome and on management decisions.

\section{Authors' affiliations \\ T D Sudarsanam, L e Jeyaseelan, K Thomas, G John, CMC, Vellore, India}

Funding: none.

Conflicts of interest: none declared.

\section{REFERENCES}

1 Debidas R. Experience with mobile assisted ventilatory care: an analysis over 15 years. Lung India 1997;XV:173-7.

2 Eapen CE, Thomas K, Cherian AM, et al. Predictors of mortality in a medical intensive care unit. Natl Med J India 1997;10:270-2.

3 Knaus WA, Draper EA, Wagner DP, et al. Prognosis in acute organ system failure. Ann Surg 1985;202:685-93.

4 Knaus WA, Draper EA, Wagner DP, et al. APACHE II: A severity of disease classification system. Crit Care Med 1985;13:818-29.

5 Knaus WA, Douglas DP, Draper EA, et al. The APACHE III prognostic system: Risk prediction of mortality for critically ill hospitalized adult patients. Chest 1991; 100:1619-36.
6 McNeil BJ, Keeler E, Adelstein SJ. Primer on certain elements of decisionmaking. N Engl J Med 1975;293:211-15.

7 Weiss SM, Hudson LD. Outcome from respiratory failure. Crit Care Clin 1994; 10:197-213.

8 Peters SG, Meadows JA, Gracey DR. Outcome of respiratory care. 3rd ed. Philadelphia: Lea and Febiger, 1982:328.

9 Jeffrey AA, Warren PM, Flenley DC. Acute hypercapnic respiratory failure in patients with chronic obstructive lung disease; risk factors and use of guidelines for management. Thorax 1992;47:34.

10 Menzies R, Gibbons W, Goldberg P. Determinants of weaning and survival among patients with COPD who required mechanical ventilation for acute respiratory failure. Chest 1989;95:398.

11 Spicher JE, White DP. Outcome and function following prolonged mechanical ventilation. Arch Intern Med 1987; 147:421-5.

12 Ludwigs UG, Baehrendtz S, Wanecek $M$, et al. Mechanical ventilation in medical and neurological disease: 11 years of experience. J Intern Med 1991;229:117-24

13 Portier F, Defouilloy C, Muir JF. Determinants of immediate survival among chronic respiratory insufficiency patients admitted to an intensive care unit for acute respiratory failure. A prospective multicentre study. The French task group for acute respiratory failure. A prospective multicentre study. Chest group for acute respir:

14 Sloane PJ, Gee MH, Gottleib JE, et al. A multicentric registry of patients with acute respiratory distress syndrome. Physiology and outcome. Am Rev Respir Dis 1992;146:419-26.

15 Suchyta MR, Klemmer Tp, Elliot CG, et al. The adult respiratory distress syndrome: a report of survival and modifying factors. Chest 1992;101:1074-9.

16 Miller EJ, Cohen AB, Nagao S, et al. Elevated levels of NPA-1/interluekin-8 are present in the air spaces of patients with the adult respiratory distress syndrome and are associated with increased mortality. Am Rev Respir Dis 1992;146:427-32.

17 Hudson LD. Survival data in patients with acute and chronic lung disease requiring mechanical ventilation. Am Rev Respir Dis 1989;140:S19-24

18 Gupta D, Ramanathan RP, Aggarwal AN, et al. Assessment of factors predicting outcome of acute respiratory distress syndrome in North India. Respirology $2001 ; 6: 125-30$.

19 Petty TL. Intensive and rehabilitatory care. 3rd ed. Philadelphia: Lea and Febiger, 1982:328

20 Peters Jl, Bell RC, Riphoda TJ, et al. Clinical determinants of abnormalities in pulmonary functions in survivors of the adult respiratory distress syndrome. Am Rev Respir Dis 1989;139:1 163-8.

21 Nevins ML, Epstein SK. Predictors of outcome for patients with COPD requiring invasive mechanical ventilation. Chest 2001;119:1840-9.

22 Diener CF, Burrows B. Further observations on the course and prognosis of chronic obstructive lung disease. Am Rev Respir Dis 1975;111:719.

23 Kettel $\mathrm{L}$, Diener CF, Morse JO, et al. Treatment of acute respiratory acidosis in chronic obstructive lung disease. JAMA 1971;217:1503-5.

24 Warren PM, Flenley DC, Miller JS, et al. Respiratory failure revisited; acute exacerbations of chronic bronchitis between 1961-68 and 1970-76. Lancet 1989;i:467-70. 